\title{
Clinical application of RapidArc volumetric modulated arc therapy as a component in whole brain radiation therapy for poor prognostic, four or more multiple brain metastases
}

\author{
Seung Heon Lee, MD¹, Kyu Chan Lee, MD', Jinho Choi, MS', Hye Young Kim, MS'1, \\ Seok Ho Lee, MD'1, Ki Hoon Sung, MD¹, Yunmi Kim, PhD² \\ Departments of ${ }^{1}$ Radiation Oncology, ${ }^{2}$ Nursing, Gachon University Gil Hospital, Incheon, Korea
}

Purpose: To determine feasibility of RapidArc in sequential or simultaneous integrated tumor boost in whole brain radiation therapy (WBRT) for poor prognostic patients with four or more brain metastases.

Materials and Methods: Nine patients with multiple $(\geq 4)$ brain metastases were analyzed. Three patients were classified as class II in recursive partitioning analysis and 6 were class III. The class III patients presented with hemiparesis, cognitive deficit, or apraxia. The ratio of tumor to whole brain volume was 0.8-7.9\%. Six patients received 2-dimensional bilateral WBRT, (30 Gy/1012 fractions), followed by sequential RapidArc tumor boost (15-30 Gy/4-10 fractions). Three patients received RapidArc WBRT with simultaneous integrated boost to tumors (48-50 Gy) in 10-20 fractions.

Results: The median biologically effective dose to metastatic tumors was $68.1 \mathrm{~Gy}_{10}$ and $67.2 \mathrm{~Gy}_{10}$ and the median brain volume irradiated more than $100 \mathrm{~Gy}_{3}$ were $1.9 \%\left(24 \mathrm{~cm}^{3}\right)$ and $0.8 \%\left(13 \mathrm{~cm}^{3}\right)$ for each group. With less than 3 minutes of treatment time, RapidArc was easily applied to the patients with poor performance status. The follow-up period was 0.3-16.5 months. Tumor responses among the 6 patients who underwent follow-up magnetic resonance imaging were partial and stable in 3 and 3 , respectively. Overall survival at 6 and 12 months were $66.7 \%$ and $41.7 \%$, respectively. The local progression-free survival at 6 and 12 months were $100 \%$ and $62.5 \%$, respectively.

Conclusion: RapidArc as a component in whole brain radiation therapy for poor prognostic, multiple brain metastases is an effective and safe modality with easy application.

Keywords: Multiple brain metastases, Poor prognostic, RapidArc, Volumetric modulated arc therapy

\section{Introduction}

The number of patients with brain metastasis is increasing as the survival time of cancer patients are extended with modern therapeutic measures. About 20-40\% of whole cancer patients are estimated to develop brain metastasis and the most common primary sites are lung, breast, and gastrointestinal cancer $[1,2]$. Reportedly, when brain metastases were first diagnosed in the solid tumor patients, single metastasis were $37-50 \%$, and multiple were 50-63\% [3-5]. Whole brain

Received 6 March 2012, Revised 30 April 2012, Accepted 4 May 2012.

Correspondence: Kyu Chan Lee, MD, Department of Radiation Oncology, Gachon University Gil Hospital, 21 Namdongdaero 774 beon-gil, Namdong-gu, Incheon 405-760, Korea. Tel: +82-32-460-8050, Fax: +82-32-460-3009, E-mail: Kyu22@ gilhospital.com

(c) This is an Open Access article distributed under the terms of the Creative Commons Attribution Non-Commercial License (http://creativecommons.org/ licenses/by-nc/3.0/) which permits unrestricted non-commercial use, distribution, and reproduction in any medium, provided the original work is properly cited.

www.e-roj.org 
radiation therapy (WBRT) has been a mainstay of the treatment for the patients with brain metastasis. Unfortunately, median survival time following WBRT alone was 3-6 months regardless of primary tumor histology [6-11]. More than 50\% of the patients will develop local recurrence at the original brain metastasis after WBRT alone [12]. In single brain metastasis, surgical removal of tumor followed by WBRT significantly lowered the local recurrence in the original site.

In the patients with multiple brain metastases of 4 or more lesions, surgical approach is limited. Most patients in this category have poor performance status and other poor prognostic factors. In these patients, stereotactic radiosurgery (SRS) is not easily applicable. Furthermore, SRS with or without WBRT have technical or dosimetric problems concerning the total irradiation dose to normal brain tissue. One study concluded that the irradiated dose to brain was acceptable when the number of targets was 7 or less in case of Gamma knife (Elekta AB, Stockholm, Sweden) SRS [13].

RapidArc is a volumetric intensity modulated arc therapy. It can be used to give ablative high dose to tumor target only in the brain metastases and can also be used in simultaneous WBRT and tumor boost. Simultaneous RapidArc WBRT and boost to metastatic tumors was compared to simple summation of sequential 2-dimensional (2D) WBRT and radiosurgery boost in patients with one to three brain metastases [14]. Simultaneous RapidArc WBRT and tumor boost plans showed much steeper dose gradients outside the metastatic lesion in the brain than plans with simple summation of sequential 2D WBRT and radiosurgery. This phenomenon, resulting from the modulation of the WBRT dose within the area of the boost dose gradient, gives possibility for its more broad application to more advanced, more multiple brain metastases. However, clinical feasibility concerning the amount of boost dose to tumors and whole brain for the patients with more than 4 lesions is not established yet.

The purpose of this study is to determine the feasibility of RapidArc volumetric modulated arc therapy as a component in whole brain radiation therapy in the treatment for the patients with poor prognostic, four or more multiple brain metastases and to report its early clinical results.

\section{Materials and Methods}

Nine patients with 4 or more brain metastases, treated between April 2010 and February 2011, were included in this retrospective analysis. The median age was 59 years (range, 48 to 76 years) and 3 patients was over 65 years (Table 1). Primary cancer sites were lung ( 6 patients), breast ( 2 patients) and uterine cervix (1 patient). According to recursive partitioning analysis (RPA) classification, 3 patients were class II and 6 were III. Patients of class III had various kinds of moderate to severe neurologic symptoms such as hemiparesis, cognitive deficit, apraxia or hallucination. Recently, Sperduto et al. [15] reported the diagnosis specific graded prognostic assessment (DS-GPA) indices to estimate the survival. According to DS-GPA score, all 5 patients of non-small cell lung cancer primary were scored 0-1 and one patient of small cell lung cancer primary was 1.5. Two patients of breast cancer primary were scored 2.0.

Table 1. Patient characteristics

\begin{tabular}{cccccccccc}
\hline $\begin{array}{c}\text { Patient } \\
\text { no. }\end{array}$ & Sex & Age (yr) & $\begin{array}{c}\text { RPA } \\
\text { class }\end{array}$ & $\begin{array}{c}\text { DS-GPA } \\
\text { score }\end{array}$ & $\begin{array}{c}\text { Primary } \\
\text { cancer }\end{array}$ & $\begin{array}{c}\text { Maximum } \\
\text { tumor size } \\
(\mathrm{cm})\end{array}$ & $\begin{array}{c}\text { No. of } \\
\text { tumor }\end{array}$ & $\begin{array}{c}\text { Tumor } \\
\text { volume } \\
\left(\mathrm{cm}^{3}\right)\end{array}$ & $\begin{array}{c}\text { Volume } \\
\text { ratio } \\
(\%)\end{array}$ \\
\hline 1 & F & 62 & II & 0.5 & NSCLC & 4.5 & 10 & 69.2 & 6.3 \\
2 & F & 53 & III & 2.0 & Breast cancer & 3.8 & 5 & 79.5 & 6.4 \\
3 & M & 76 & III & 0 & NSCLC & 3.3 & 5 & 44.9 & 3.3 \\
4 & M & 68 & III & 0 & NSCLC & 3.6 & 6 & 73.8 & 5.3 \\
5 & F & 48 & II & 2.0 & Breast cancer & 2 & 9 & 28.4 & 2.2 \\
6 & M & 59 & III & 1.5 & SCLC & 3.1 & 6 & 35.0 & 2.3 \\
7 & M & 51 & II & 1 & NSCLC & 2.9 & 9 & 70.4 & 5.2 \\
8 & F & 52 & III & $-{ }_{\text {b) }}$ & Cervix cancer & 4.5 & 4 & 106.0 & 7.9 \\
9 & M & 66 & III & 0.5 & NSCLC & 3 & 8 & 13.6 & 0.8 \\
\hline
\end{tabular}

RPA, recursive partitioning analysis; DS-GPA, diagnosis-specific graded prognostic assessment; NSCLC, non-small cell lung cancer; SCLC, small cell lung cancer.

${ }^{\text {a) }}$ olume ratio = tumor volume / whole brain volume. ${ }^{\text {b) }}$ Cervix cancer was not defined in DS-GPA index. 
Neurocognitive function test could not be done in the patients with poor general condition. The number of brain metastases was 4-10 and the maximum diameter of the metastatic lesions ranged from 2.0 to $4.5 \mathrm{~cm}$. The tumor volume was $14-106 \mathrm{~cm}^{3}$ (median, $69 \mathrm{~cm}^{3}$ ) and the ratio of tumor to whole brain volume was $0.8-7.9 \%$.

Thin slice magnetic resonance imaging (MRI) and computer tomography (CT) images, $1 \mathrm{~mm}$ without gap, were fused to delineate the targets and other normal structures including organs at risk (OAR) on iPlan software v4.1 (BrainLab, Munich, Germany). Gross tumor volume (GTV) was determined based on the T1 gadolinium enhanced MRI image. Planning target volume (PTV) was derived from GTV with 1-2 mm margin considering tumor location and nearby OAR. The contoured target and organ structures were transferred to the Eclipse treatment planning system 8.6.1 (Varian Medical Systems, Palo Alto, CA, USA), for RapidArc planning.
Treatment plans were generated with measured beam data set of $6 \mathrm{MV}$ photons of Novalis Tx (Varian Medical Systems) equipped with the $2.5 \mathrm{~mm}$ thickness high-definition 120 multileaf collimator (HD 120 MLC; Varian Medical Systems). To achieve the desired level of modulation required in RapidArc, several parameters such as isocenter, field size, gantry rotation angle and collimator angle were adjusted for target shape and location. Angle of collimator was set to orthogonal position to decrease the tongue-and-groove effect. Double arc $\left(719.6^{\circ}\right)$ plans were used in 8 patients and triple arc $\left(989.6^{\circ}\right)$ in 1. Dose calculations were performed by use of the anisotropic analytical algorithm calculation model, with calculation grid size of $2.5 \mathrm{~mm}$, and with tissue heterogeneity correction. Fig. 1 shows a result of RapidArc plan for sequential tumor boost after 2D WBRT to give 25 Gy to tumors in 10 fractions. Fig. 2 shows the RapidArc WBRT plan with simultaneous integrated tumor boost. In this case, 48 Gy to tumor and 24 Gy to brain
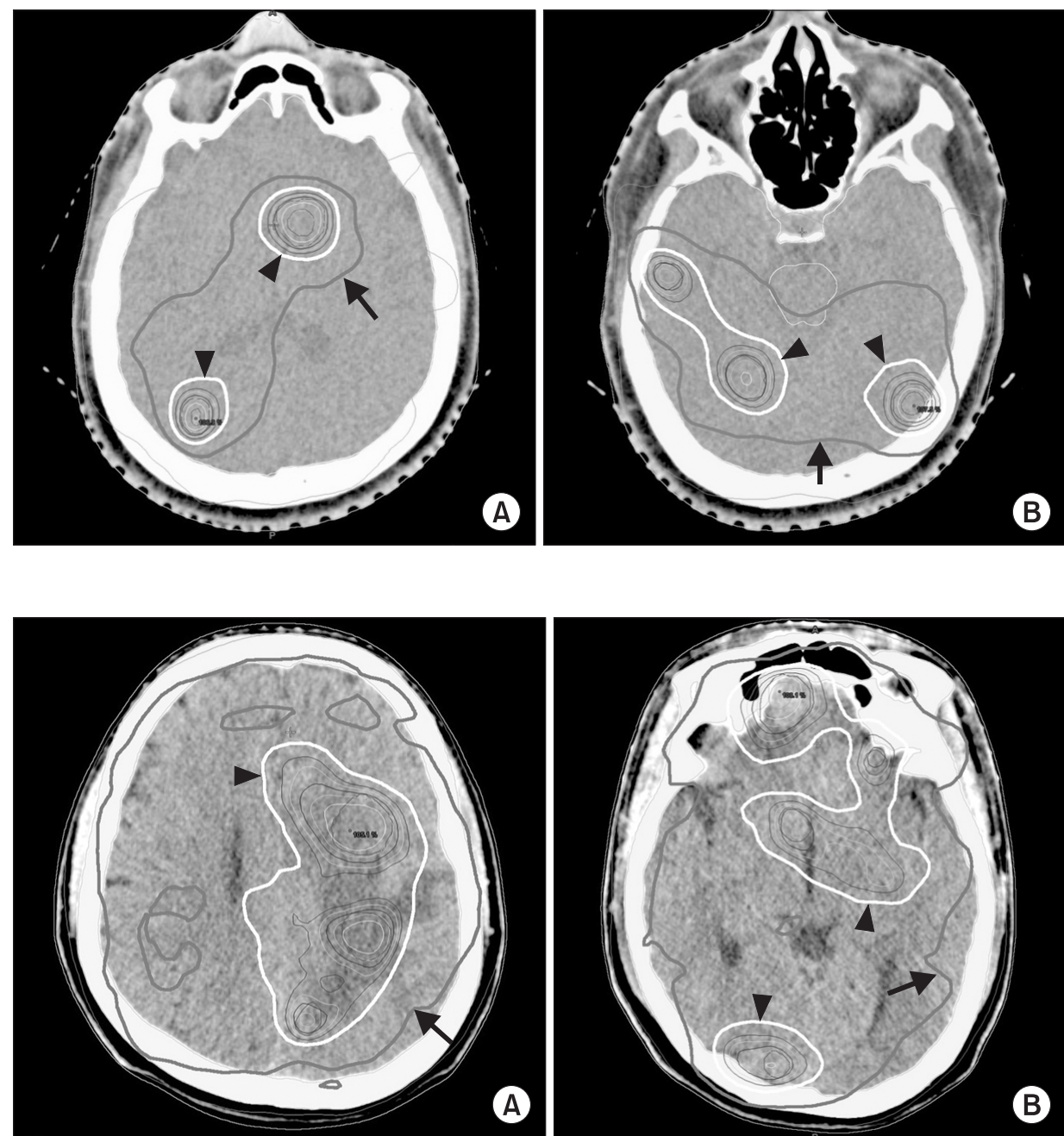

Fig. 2. RapidArc whole brain radiation therapy plan with simultaneous integrated tumor boost for patient no. 7, who had 9 targets. (A) and (B) are CT images of different levels of the brain. The gray lines (arrow) show the $50 \%$ isodose line and cover $88.9 \%$ of the whole brain. The white lines (arrowhead) here reveal the $70 \%$ isodose line. 

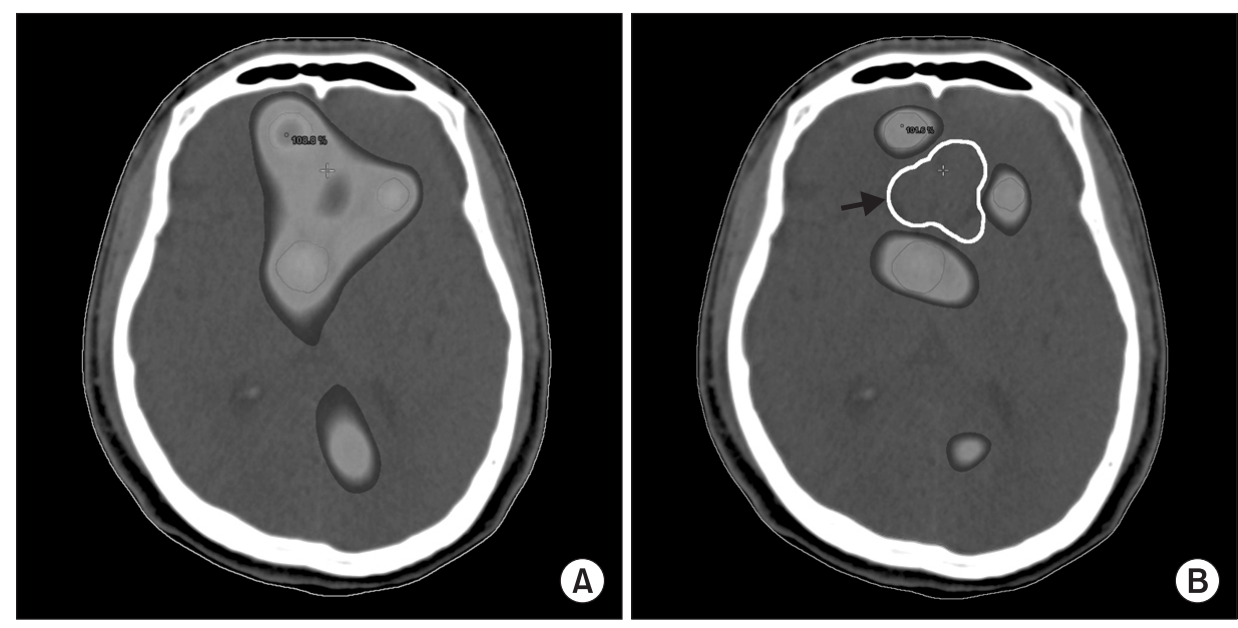

Fig. 3. Initial RapidArc plan for the patient no. 7. The color painted area is receiving more than $80 \%$ of prescription dose (A). Among the 3 targets visible, avoidance area (white line, arrow) is depicted to prevent unnecessary high dose in normal brain (B). After re-plan with avoidance, dose optimization was done and unintentional high dose among the targets are decreased.

Table 2. Treatment group and prescription dose

\begin{tabular}{|c|c|c|c|c|c|c|}
\hline Radiotherapy method & Patient no. & WBRT (Gy/fx) & RA boost (Gy/fx) & $\begin{array}{l}\text { RA WBRT with } \\
\text { SIB (Gy:Gy) } / \text { fx }\end{array}$ & $\begin{array}{c}\text { BED to } \\
\text { tumor }\left(G y_{10}\right)\end{array}$ & $\begin{array}{l}\text { Median BED to } \\
\text { tumor }\left(\mathrm{Gy}_{10}\right)\end{array}$ \\
\hline \multirow{6}{*}{$\begin{array}{l}\text { Conventional WBRT + RA } \\
\text { boost to tumor (Sequential } \\
\text { boost group) }\end{array}$} & 1 & $30 / 12$ & $15 / 6$ & - & 56.3 & \multirow[t]{6}{*}{68.1} \\
\hline & 2 & $30 / 12$ & $20 / 8$ & - & 62.5 & \\
\hline & 3 & $30 / 12$ & $20 / 4$ & - & 67.5 & \\
\hline & 4 & $30 / 12$ & $24 / 8$ & - & 68.7 & \\
\hline & 5 & $30 / 12$ & $25 / 10$ & - & 68.8 & \\
\hline & 6 & $30 / 10$ & $30 / 10$ & - & 78.0 & \\
\hline \multirow{3}{*}{$\begin{array}{l}\text { WBRT with SIB by RA } \\
\text { (Simultaneous boost group) }\end{array}$} & 7 & - & - & $(24: 48) / 12$ & 67.2 & \multirow[t]{3}{*}{67.2} \\
\hline & 8 & - & - & $(25: 50) / 20$ & 62.5 & \\
\hline & 9 & - & - & $(25: 50) / 10$ & 75.0 & \\
\hline
\end{tabular}

2D, 2-dimensional; RA, RapidArc; WBRT, whole brain radiotherapy; SIB, simultaneous integrated boost; BED, biologically effective dose.

a) Prescription dose to (whole brain:tumor).

were prescribed in 12 fractions. If needed, avoidance area was added to make more conformal optimization (Fig. 3). Hippocampal sparing in RapidArc plan was started from June 2011 in our institution, that for the patients in the current study it was not performed.

Lying on the treatment couch, the patient's head was immobilized with Type-S head thermoplastic mask (CIVCO, Kalona, IA, USA) or BrainLab Frameless SRS mask (BrainLab). To ensure the accuracy, ExacTrac patient positioning system (BrainLab) was used to set up the patient as a first step. Then, cone beam CT image was obtained before the treatment to reconfirm the target position. Treatment beam-on time was about 3 minutes or less (2.50-3.04) in all cases.

Six patients out of 9 , received conventional 2D WBRT (30 Gy/10-12 fractions), followed by sequential RapidArc tumor boost (15-30 Gy/4-10 fractions) in 2-5 weeks after WBRT (Sequential boost group) (Table 2). Boost doses and fraction sizes were determined depending on the number and location of the metastatic tumors. Biologically effective dose (BED) calculation based on linear-quadratic model was used for plan comparison assuming that $\alpha / \beta$ of tumor and normal brain as 10 and 3 , respectively. In this article, Gy ${ }_{10}$ means BED of tumor and $\mathrm{Gy}_{3} \mathrm{BED}$ of brain. In sequential boost group, BED was simply summed without considering time factor. Total prescription dose to tumors ranged from 56.3 to $78.0 \mathrm{~Gy}_{10}$ (median, $68.1 \mathrm{~Gy}_{10}$ ). Three patients received RapidArc WBRT with simultaneous integrated boost to tumors (48-50 Gy) in 10-20 fractions (Simultaneous boost group). Total dose prescribed for the tumor ranged from 62.5 to $75 \mathrm{~Gy}_{10}$ (median, $\left.67.2 \mathrm{~Gy}_{10}\right)$

Follow-up MRIs were checked at 1-3 months after completion of the treatment for response evaluation in 6 patients. Tumor response was assessed using Response Evaluation Criteria in Solid Tumors (RECIST) ver. 1.1. 
Table 3. Summary of total biologically effective dose to whole brain and high dose volume

\begin{tabular}{lcc}
\hline & Sequential boost group (median) & Simultaneous boost group (median) \\
\hline Mean brain BED $\left(\mathrm{Gy}_{3}\right)$ & $61.4-72.0(65.1)$ & $46.2-57.0(52.5)$ \\
Maximum brain BED $\left(\mathrm{Gy}_{3}\right)$ & $90.6-127.7(112.4)$ & $102.0-136.8(131.2)$ \\
$V_{100 G y 3}$ & $\left.0-56 \mathrm{~cm}^{3}\left(24 \mathrm{~cm}^{3}\right)^{\mathrm{a}}\right), 0-3.7 \%(1.9 \%)^{b)}$ & $3-55 \mathrm{~cm}^{3}\left(13 \mathrm{~cm}^{3}\right), 0.2-4.2 \%(0.8 \%)$ \\
$V_{120 \mathrm{y} 3}$ & $0-5 \mathrm{~cm}^{3}\left(0 \mathrm{~cm}^{3}\right), 0-0.3 \%(0.0 \%)$ & $0-5 \mathrm{~cm}^{3}\left(4 \mathrm{~cm}^{3}\right), 0-0.3 \%(0.3 \%)$ \\
\hline
\end{tabular}

$\mathrm{BED}$, biologically effective dose; $V_{100 \mathrm{G} y 3}$, brain volume irradiated more than $\mathrm{BED}$ of $100 \mathrm{~Gy}_{3} ; \mathrm{V}_{120 \mathrm{G} y 3}$, brain volume irradiated more than BED of $120 \mathrm{~Gy}_{3}$.

${ }^{a)}$ Absolute brain volume irradiated more than BED of $100 \mathrm{~Gy}_{3} \cdot{ }^{b}$ Ratio of irradiated brain volume to whole brain volume.

\section{Results}

Total BED to whole brain and high dose volume are summarized in Table 3. In comparison to sequential boost group, simultaneous boost group showed lower mean dose to brain (median, $65.1 \mathrm{~Gy}_{3}$ vs. $52.5 \mathrm{~Gy}_{3}$ ), though the total tumor dose was similar in both group (median, $68.1 \mathrm{~Gy}_{10}$ vs. $67.2 \mathrm{~Gy}_{10}$ ). The brain volumes irradiated more than $100 \mathrm{~Gy}_{3}\left(\mathrm{~V}_{1006 \mathrm{y}}\right)$ were 0-56 $\mathrm{cm}^{3}$ (median, $24 \mathrm{~cm}^{3}$ ) and 3-55 $\mathrm{cm}^{3}$ (median, $13 \mathrm{~cm}^{3}$ ), respectively. The ratio of irradiated volumes to whole brain volumes were 0-3.7 \% (median, 1.9\%) and 0.2-4.2\% (median, $0.8 \%)$, respectively.

One patient died at 9 days after the completion of the radiotherapy because of respiratory failure. The median overall survival was 9 months with the follow-up periods of 0.3 16.5 months. Overall survival at 6 and 12 months were 66.7\% and $41.7 \%$, respectively (Fig. 4). Five (55.6\%) patients died of extracranial disease progression, mainly with respiratory or liver failure. The median local progression free survival was not reached and local progression free survival at 6 and 12 months was $100.0 \%$ and $62.5 \%$, respectively.

Tumor responses among the 6 patients who underwent follow-up MRI at 1-3 months after treatment were partial and stable in 3 and 3, respectively. Two patients in simultaneous boost group (no. 7 and 8) showed the regrowth of treated lesion at 7.4 and 8.2 months after treatment, without any new intracranial metastasis. The prescription doses of these 2 patients were $67.2 \mathrm{~Gy}_{10}$ and $62.5 \mathrm{~Gy}_{10}$.

After treatment, significant improvements of neurologic symptoms such as hemiparesis, cognitive deficit, hallucination or apraxia were noted in all patients. For the treatment related toxicity, grade 1 pruritus on scalp was seen in one patient. No toxicity of grade 3 or above was found during or after treatment.

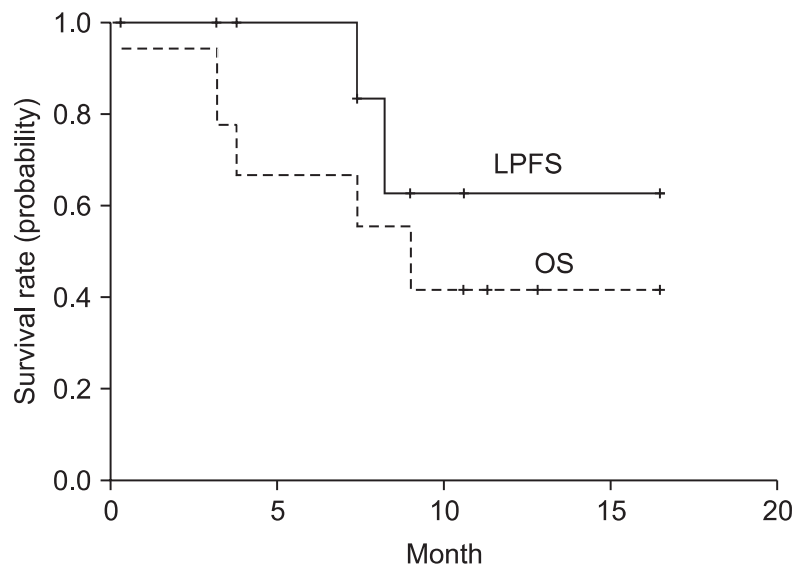

Fig. 4. Overall survival (OS) and local progression free survival (LPFS) rate for all patients.

\section{Discussion and Conclusion}

Whole brain radiotherapy has long been a standard treatment for patients with brain metastasis. The treatment result of brain metastasis with WBRT alone was, however, generally poor with median survival in the range of 3-6 months [610,12]. Radiobiologically, 30 Gy in 10 fractions to a solid tumor is not sufficient to achieve long-term tumor control [16]. Considerable efforts have been made to explore additional or alternative treatment modalities that have the potential to improve local tumor control.

In patients with good performance status and limited extracranial disease, surgical resection followed by post-operative WBRT showed a superior treatment results compared to WBRT alone for single brain metastasis [17]. For single brain metastasis, many studies reported that SRS with or without WBRT resulted in almost equal survival rates with surgical resection followed by WBRT [18-21]. Radiation Therapy Oncology Group (RTOG) 95-08 trial studied WBRT with or without SRS for the patients with one to three brain metastases [10]. The risk of developing a local recurrence was 
$43 \%$ greater with WBRT alone $(p=0.0021)$. WBRT and SRS boost improved intracranial local control rates and Karnofsky Performance Status for all patients. Survival gain, however, was demonstrated in single metastasis patients. Kondziolka et al. [22] randomized patients with 2-4 solid metastatic brain tumors (each $\leq 2.5 \mathrm{~cm}$ in mean diameter). A significant improvement in the rate of local tumor control was found after WBRT plus radiosurgery in comparison to WBRT alone. The rate of local failure at 1 year was 100\% after WBRT alone, but only $8 \%$ at 1 year in surviving patients who had boost radiosurgery.

For the patients with 4 or more metastatic brain tumors, however, it is difficult to perform surgical resection. SRS also has difficulties in its clinical application and planning process because of high cumulative dose to whole brain. Takahashi et al. [13] suggested the practical limit of Gamma knife (Elekta $A B)$ SRS in terms of the number of SRS targets based on the irradiation dose to the brain. Given a target surface dose of $25 \mathrm{~Gy}$ in single session SRS, the volume of the brain irradiated to a dose of 5 Gy or higher was increased with the increase of the number of targets. When the number of the targets exceeded 7, more than 50\% of the entire brain was irradiated at least $8.7 \mathrm{~Gy}$. The increase of irradiation dose to brain, by the number of targets, is mainly due to the overlapping dose of each beam for each target. As the number of targets increases and multiple isocenters are used, more beam overlapping is inevitable in Gamma Knife (Elekta AB), CyberKnife (Accuray, Sunnyvale, CA, USA) or linac based SRS. On the other hand, every target is considered simultaneously in RapidArc and the dose to brain is minimized by the optimization process even when the isocenter is not single.

Lawrence et al. [23] reviewed radiation-induced brain injury in fractionated radiotherapy with fraction sizes of 2.5 Gy or more. Radiation necrosis usually occurred in the range of 100$120 \mathrm{~Gy}_{3}$, although the incidence and severity of toxicity was unpredictable because the data were too scattered to allow plotting of "best-fit" line. In the current study, the brain volume irradiated more than $100 \mathrm{~Gy}_{3}\left(\mathrm{~V}_{100 \mathrm{~Gy}}\right)$ was 0-4.2\% (median, $0.9 \%$ ) in all patients. No radiation necrosis was observed on follow MRIs until now and no significant treatment related toxicity was found yet. A clinical trial is ongoing to find tolerability of RapidArc WBRT and simultaneous boost in brain metastases (clinicaltrials.gov identifier NCT01218542). There are 4 study cohorts starting from giving 25 Gy in 10 fractions to the whole brain as a prophylactic dose without simultaneous infield boost (SIB) to gross disease (cohort baseline). SIB dose escalation will be up to $3.5 \mathrm{~Gy}$ per fraction (cohort 3, total tumor dose of $60 \mathrm{~Gy} / 10$ fractions). Median dose of current study is in between cohort group 1 and 2 . In simultaneous boost group, considering shortened overall treatment time and occasional areas of small foci of high dose spots, median WBRT prescription dose was reduced to $25 \mathrm{~Gy}$ in 10 fractions. While mean dose to brain was reduced from 65.1 $\mathrm{Gy}_{3}$ to $52.5 \mathrm{~Gy}_{3}$ in simultaneous boost group, total irradiation dose to tumor was similar in both groups (median, $68.1 \mathrm{~Gy}_{10}$ and $67.2 \mathrm{~Gy}_{10}$ ) (Fig. 5). Since there was no new lesion in followup MRIs, 25 Gy in 10 fractions is considered appropriate.

Short treatment time is critical to treat the patient with
A

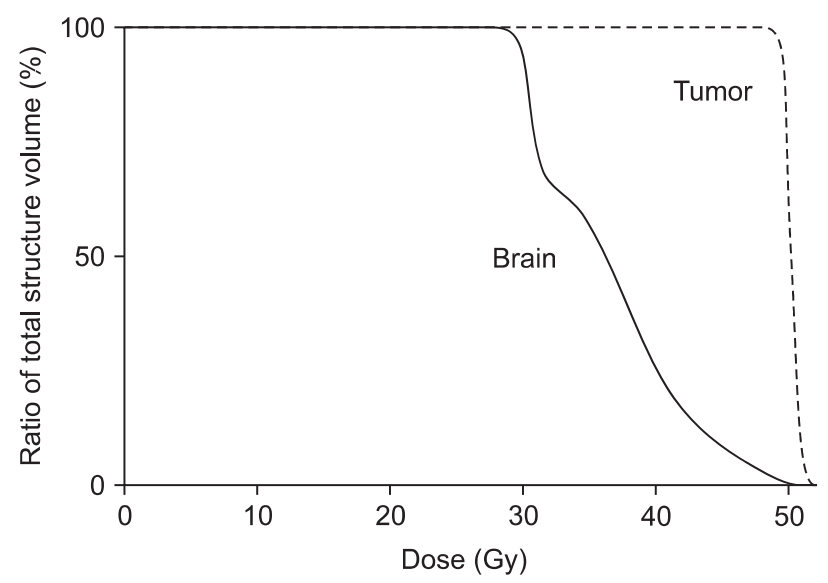

B

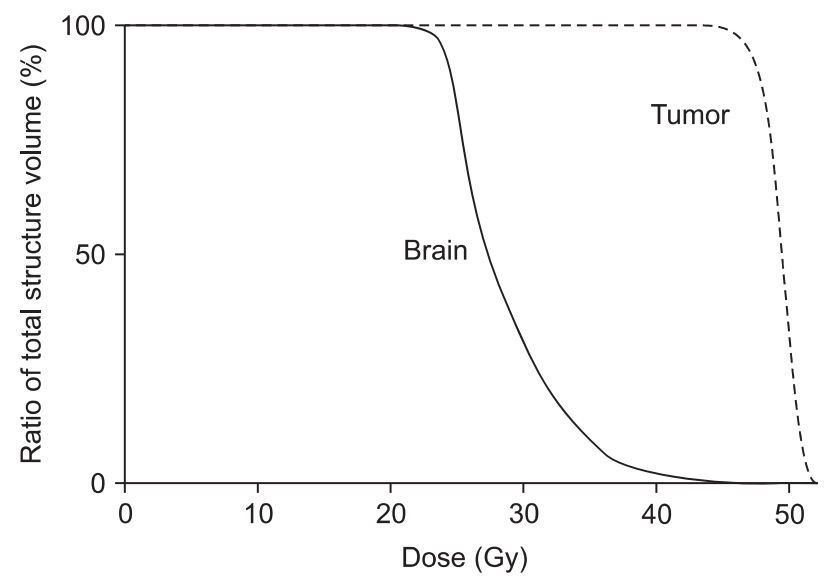

Fig. 5. Cumulative dose volume histogram of conventional whole brain radiation therapy (WBRT) with sequential RapidArc boost for patient no. 2 (A), and RapidArc WBRT with simultaneous integrated boost to tumor for patient no. 9 (B). Whole brain dose is lowered in simultaneous boost group, maintaining the similar tumor dose. Note steeper dose gradient for brain above $30 \mathrm{~Gy}$ in (B) than in (A). 
poor performance status who cannot tolerate long treatment time. Wolff et al. [24] reported that treatment time for single target SRS using conformal arc therapy was definitely longer compared to RapidArc treatment (median, 34.4 minutes vs. 4.5 minutes). For multiple targets, treatment time might be multiplied by the number of isocenters in SRS, up to one and half hours or more. However, it is not the case in RapidArc, where total treatment time is related to total arc angle. Beam on time of RapidArc treatment in the current study was about 3 minutes or less regardless of target numbers.

The overall treatment period of sequential boost group were about 6-8 weeks with 2-5 weeks interval between 2D WBRT and RapidArc boost which consisted 16-22 fractions (median, 20 fractions) in total. For RapidArc WBRT with simultaneous boost group, it was 2-3 weeks in 10-20 fractions. Considering relatively poor prognosis of the most patients and short estimated life span, shorter treatment period with simultaneous boost seems to be more desirable. At the initial stage of the application of RapidArc for the treatment of multiple brain metastases in our institution, sequential boost was routinely used. Currently, more patients are treated with RapidArc WBRT and simultaneous boost. In some patients with irritability or very poor performance status, sequential 2D WBRT with waiting interval gives chance to perform more elaborate treatment technique.

Although survival gain of intense local treatment, such as surgical resection or SRS, for patients with up to 3 brain metastases was reported, it has been known that remission of brain metastases or administration of more than $30 \mathrm{~Gy}$ was not associated with increased survival for the patients with more than 4 brain metastases $[25,26]$. However, Li et al. [27] reported that local progression of brain metastases deteriorated neurocognitive function and consequently the patient's quality of life. In our study, the two patients who showed tumor regrowth suffered from weakness, seizure, and mental change afterwards. The local tumor control in these patients is considered as a worthwhile treatment goal. The maximum tolerated dose of brain and optimal dose of tumors in multiple brain metastases are not established yet. Previous mentioned study, NCT01218542 (ClinicalTraials.gov) sponsored by Emory University, will hopefully reveal the possibility.

There was no RPA class I patent in the current study. Three patients were class II and 6 were class III. The median survival was 9 and 7 months, respectively. Reportedly, the median survival of RPA class II and III were 4.2 and 2.3 months [9]. Sperduto et al. [15] suggested the DS-GPA indices to estimate
Table 4. Comparison of median survival for lung and breast cancer patients by DS-GPA score

\begin{tabular}{lcccc}
\hline & \multicolumn{3}{c}{ DS-GPA score } \\
\cline { 2 - 5 } & \multicolumn{2}{c}{$0-1.0$} & \multicolumn{2}{c}{$1.5-2.0$} \\
\hline Primary cancer & Lung & Breast & Lung & Breast \\
Patient ${ }^{\text {a }}$ & 5 & - & 1 & 2 \\
Median survival (mo) & 7.40 & - & 3.20 & 12.75 \\
Estimated survival (mo) $^{\text {b) }}$ & 3.02 & 3.35 & 5.49 & 7.70 \\
\hline
\end{tabular}

DS-GPA, diagnosis-specific graded prognostic assessment.

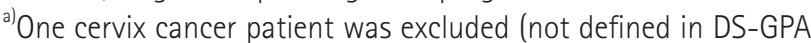

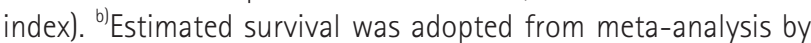
Sperduto et al. [15].

the survival and proposed prognostic index by primary tumor diagnosis. For each diagnosis, a robust separation into different GPA scores was discerned and classified into 4 groups; 0-1.0, 1.5-2.0, 2.5-3.0, and 3.5-4.0. According to DSGPA classification, current study patients were stratified to evaluate and compare early treatment results (Table 4). Eight patients were 2.0 or below, excluding one patient with cervix cancer, which was not defined in DS-GPA classification. Five patients with lung cancer primary were classified as 0-1.0 and their median survival was 7.40 months, which is much longer than that of 3.02 months estimated in the report of Sperduto et al. [15].

The current study has many limitations because it is basically a retrospective analysis, and the patient numbers are too small to make any confident conclusions. However, the early results suggests the possibility of improved quality of life for the patients with poor prognostic, four or more multiple brain metastases. In selected patients, survival might be extended with effective local control of the multiple brain metastases. To confirm this possibility, prospective study with tumor dose escalation is needed. The authors are preparing to open the prospective study including more sophisticated neurologic examinations and neurocognitive function tests before and after treatment.

In conclusion, RapidArc as a component in whole brain radiation therapy for poor prognostic, multiple brain metastases is an effective and safe modality with easy application. Considering the optimal dose to whole brain and short treatment time and reduced overall treatment period, WBRT with simultaneous boost is more desirable treatment modality. 


\section{Conflict of Interest}

No potential conflict of interest relevant to this article was reported.

\section{Acknowledgments}

This study was supported by research grant program of Gachon University.

\section{References}

1. Walker $A E$, Robins $M$, Weinfeld FD. Epidemiology of brain tumors: the national survey of intracranial neoplasms. Neurology 1985;35:219-26.

2. Silverberg $E_{1}$ Lubera JA. Cancer statistics, 1988. CA Cancer J Clin 1988;38:5-22.

3. Deutsch M, Parsons JA, Mercado R Jr. Radiotherapy for intracranial metastases. Cancer 1974;34:1607-11.

4. Delattre JY, Krol G, Thaler HT, Posner JB. Distribution of brain metastases. Arch Neurol 1988;45:741-4.

5. Gavrilovic IT, Posner JB. Brain metastases: epidemiology and pathophysiology. J Neurooncol 2005;75:5-14.

6. Horton J, Baxter DH, Olson KB. The management of metastases to the brain by irradiation and corticosteroids. Am J Roentgenol Radium Ther Nucl Med 1971;111:334-6.

7. Vecht CJ, Haaxma-Reiche H, Noordijk EM, et al. Treatment of single brain metastasis: radiotherapy alone or combined with neurosurgery? Ann Neurol 1993;33:583-90.

8. Mintz AH, Kestle J, Rathbone MP, et al. A randomized trial to assess the efficacy of surgery in addition to radiotherapy in patients with a single cerebral metastasis. Cancer 1996;78:1470-6.

9. Gaspar L, Scott C, Rotman M, et al. Recursive partitioning analysis (RPA) of prognostic factors in three Radiation Therapy Oncology Group (RTOG) brain metastases trials. Int J Radiat Oncol Biol Phys 1997;37:745-51.

10. Andrews DW, Scott CB, Sperduto PW, et al. Whole brain radiation therapy with or without stereotactic radiosurgery boost for patients with one to three brain metastases: phase III results of the RTOG 9508 randomised trial. Lancet 2004;363:1665-72.

11. Komarnicky LT, Phillips TL, Martz K, Asbell S, Isaacson S, Urtasun R. A randomized phase III protocol for the evaluation of misonidazole combined with radiation in the treatment of patients with brain metastases (RTOG-7916). Int J Radiat Oncol Biol Phys 1991;20:53-8.

12. Patchell RA, Tibbs PA, Walsh JW, et al. A randomized trial of surgery in the treatment of single metastases to the brain. $\mathrm{N}$
Engl J Med 1990;322:494-500

13. Takahashi M, Narabayashi I, Kuroiwa $T$, Uesugi $Y$, Tatsumi $T$, Inomata T. Stereotactic radiosurgery (SRS) for multiple metastatic brain tumors: effects of the number of target tumors on exposure dose in normal brain tissues. Int J Clin Oncol 2003;8:289-96.

14. Lagerwaard FJ, van der Hoorn EA, Verbakel WF, Haasbeek CJ, Slotman BJ, Senan S. Whole-brain radiotherapy with simultaneous integrated boost to multiple brain metastases using volumetric modulated arc therapy. Int J Radiat Oncol Biol Phys 2009;75:253-9.

15. Sperduto PW, Kased N, Roberge $D$, et al. Summary report on the graded prognostic assessment: an accurate and facile diagnosis-specific tool to estimate survival for patients with brain metastases. J Clin Oncol 2012;30:419-25.

16. Kwok Y, Patchell RA, Regine WF. Palliation of brain and spinal cord metastases. In: Halperin EC, Perez CA, Brady LW, editors. Perez and Brady's principles and practice of radiation oncology. 5th ed. Philadelphia, PA: Lippincott Williams \& Wilkins; 2008. p. 1974-85.

17. Gaspar LE, Mehta MP, Patchell RA, et al. The role of whole brain radiation therapy in the management of newly diagnosed brain metastases: a systematic review and evidence-based clinical practice guideline. J Neurooncol 2010;96:17-32.

18. Muacevic A, Wowra B, Siefert A, Tonn JC, Steiger HJ, Kreth FW. Microsurgery plus whole brain irradiation versus Gamma Knife surgery alone for treatment of single metastases to the brain: a randomized controlled multicentre phase III trial. J Neurooncol 2008;87:299-307.

19. Schoggl A, Kitz K, Reddy $M$, et al. Defining the role of stereotactic radiosurgery versus microsurgery in the treatment of single brain metastases. Acta Neurochir (Wien) 2000;142:621-6.

20. O'Neill BP, Iturria NJ, Link MJ, Pollock BE, Ballman KV, O'Fallon JR. A comparison of surgical resection and stereotactic radiosurgery in the treatment of solitary brain metastases. Int J Radiat Oncol Biol Phys 2003;55:1169-76.

21. Rades $D$, Bohlen $G$, Pluemer $A$, et al. Stereotactic radiosurgery alone versus resection plus whole-brain radiotherapy for 1 or 2 brain metastases in recursive partitioning analysis class 1 and 2 patients. Cancer 2007;109:2515-21.

22. Kondziolka D, Patel A, Lunsford LD, Kassam A, Flickinger JC. Stereotactic radiosurgery plus whole brain radiotherapy versus radiotherapy alone for patients with multiple brain metastases. Int J Radiat Oncol Biol Phys 1999;45:427-34.

23. Lawrence $Y R$, Li XA, el Naqa I, et al. Radiation dose-volume effects in the brain. Int J Radiat Oncol Biol Phys 2010;76(3 Suppl):S20-7.

24. Wolff HA, Wagner DM, Christiansen $H$, Hess CF, Vorwerk $H$. 
Single fraction radiosurgery using Rapid Arc for treatment of intracranial targets. Radiat Oncol 2010;5:77.

25. Nieder C, Andratschke N, Grosu AL, Molls M. Recursive partitioning analysis (RPA) class does not predict survival in patients with four or more brain metastases. Strahlenther Onkol 2003;179:16-20.

26. Pollock BE, Brown PD, Foote RL, Stafford SL, Schomberg PJ.
Properly selected patients with multiple brain metastases may benefit from aggressive treatment of their intracranial disease. J Neurooncol 2003;61:73-80.

27. Li J, Bentzen SM, Li J, Renschler M, Mehta MP. Relationship between neurocognitive function and quality of life after whole-brain radiotherapy in patients with brain metastasis. Int J Radiat Oncol Biol Phys 2008;71:64-70. 